\title{
Incremento de la resistencia a eritromicina de Streptococcus pneumoniae, Colombia, 1994-2008
}

\author{
Marylin Hidalgo, Claudia Santos, Carolina Duarte, Elizabeth Castañeda, Clara Inés Agudelo \\ Grupo de Microbiología, Instituto Nacional de Salud, Bogotá, D.C., Colombia
}

Introducción. Streptococcus pneumoniae es un agente comúnmente implicado en enfermedad invasora. Los macrólidos constituyen un tratamiento alternativo para las infecciones por S. pneumoniae resistente a los $\beta$-lactámicos. Sin embargo, la resistencia a macrólidos se ha incrementado a nivel mundial.

Objetivo. Determinar la frecuencia de la resistencia a la eritromicina de $S$. pneumoniae en 15 años de vigilancia y caracterizar fenotípica y genotípicamente los aislamientos resistentes.

Materiales y métodos. Se analizaron los datos demográficos de los pacientes, la sensibilidad antimicrobiana y los serotipos de los aislamientos resistentes a la eritromicina, recuperados entre 1994 y 2008. Se determinaron los fenotipos por la técnica del doble disco, y los genotipos, por PCR y PFGE. Todos los aislamientos se recuperaron de enfermedad invasiva y fueron proporcionados por los laboratorios nacionales de salud pública.

Resultados. Se recuperaron 3.241 aislamientos invasores; 136 (4,2 \%) presentaron resistencia a la eritromicina. La resistencia a la eritromicina se incrementó entre 1994-1996 y 2006-2008, de 2,4 \% a $6,9 \%$ en menores de 6 años y, de 3,3 \% a 5,7\%, en adultos.

Los serotipos más frecuentes fueron $6 \mathrm{~B}(36,8 \%), 14(16,9 \%)$ y $6 \mathrm{~A}(17,6 \%)$. El fenotipo constitutivo cMLSB se determinó en 87 aislamientos; 82 tenían el gen ermB. El fenotipo M se determinó en 46; 45 tenían el gen mefA, tres aislamientos expresaron fenotipo inducible (iMLS ${ }_{\mathrm{B}}$ ) y un aislamiento presentaba el gen ermB. Por PFGE, se determinó que 50 aislamientos estaban relacionados con clones internacionales, de los cuales, 58 \% eran España ${ }^{6 \mathrm{~B}} \mathrm{ST}$ 90, 26 \% eran España ${ }^{\vee v}$ ST156, $8 \%$ eran Colombia $23 F_{-}$ST338 y $8 \%$ eran España $23 F_{-}$ST81.

Conclusiones. Se observó incremento en la resistencia a la eritromicina, relacionada principalmente con el mecanismo de metilación ribosómica y con el clon España ${ }^{6 B}-S T 90$ que ha circulado en Colombia desde 1994.

Palabras clave: Streptococcus pneumoniae, eritromicina, resistencia a medicamentos, vigilancia, genotipo, fenotipo

Increase in erythromycin-resistant Streptococcus pneumoniae in Colombia, 1994-2008

Introduction. Streptococcus pneumoniae is a commonly implicated agent in invasive disease. For infections of $S$. pneumoniae resistant to $\beta$-lactam, macrolides are an alternative treatment. However, resistance to macrolides has increased worldwide as well.

Objective. The frequency of resistance to erythromycin was determined for $S$. pneumoniae over a 15-year surveillance period, and the resistant isolates were characterized phenotypically and genotypically.

Materials and methods. Demographic data of the patients, antimicrobial susceptibility and serotypes were analyzed for 3,241 S. pneumoniae isolates recovered between 1994 and 2008. The phenotypes were determined by the double-disc technique and genotypes by PCR (polymerase chain reaction) and PFGE (pulsed field gel electrophoresis). Isolates were recovered from invasive diseases and were provided by national public health laboratories.

Results. Of the 3,241 isolates, 136 were resistant to erythromycin. In the 12-year period between 19941996 and 2006-2008, resistance in each 2-year sampling had increased from $2.4 \%$ to $6.9 \%$ in children under 6 years and from $3.3 \%$ to $5.7 \%$ in adults.

The most common serotypes were 6B (36.8\%), 14 (16.9\%) and 6A (17.6\%). Constitutive phenotype cMLSB was determined in 87 isolates; 82 of these expressed the ermB gene. Phenotype $M$ was determined in 46 isolates; 45 had the mefA gene. An additional three isolates expressed the inducible phenotype (iMLS ${ }_{B}$ ), and one expressed the ermB gene. By PFGE, 50 of the isolates were found to be related to international clones--58\% were Spain ${ }^{6 B}-S T 90,26 \%$ Spain $^{9 \mathrm{~V}}-\mathrm{ST} 156,8 \%$ Colombia $^{23 \mathrm{~F}}-\mathrm{ST} 338$ and $8 \%$ Spain $23 F_{-S T} 81$.

Conclusion. The increase in erythromycin resistance was primarily related to the mechanism of 
ribosomal methylation. More than half the cases were congeneric with the clone Spain ${ }^{6 B}$-ST90 that has been circulating in Colombia since 1994.

Key words: Streptococcus pneumoniae, erythromycin, drug resistance, surveillance, genotype, phenotype.

Streptococcus pneumoniae es un agente etiológico comúnmente implicado en la neumonía extrahospitalaria, la meningitis bacteriana y la otitis media, en especial, en la población infantil y en las personas mayores de 65 años (1).

Los macrólidos son una alternativa terapéutica para el tratamiento de infecciones neumocócicas ocasionadas por aislamientos resistentes a $\beta$-lactámicos. Sin embargo, en algunos países de la región, el porcentaje de resistencia a los macrólidos se ha incrementado y es preocupante la propagación mundial de los aislamientos resistentes a la eritromicina (2-4).

Se ha descrito que la resistencia de $S$. pneumoniae a la eritromicina puede ser debida a cuatro mecanismos diferentes:

- bombas de eflujo, mediadas por los genes mefA/E,

- modificación del sitio blanco por metilación de la molécula de ARNr 23S, debida a los genes erm,

- mutaciones en los dominios II o V del 23S rRNA, $\mathrm{y}$

- mutaciones en las proteínas ribosómicas L4 o L22 $(5,6)$.

La resistencia mediada por metilasas afecta también a las lincosamidas, como la clindamicina y las estreptograminas de tipo $\mathrm{B}$, lo que genera un tipo de resistencia comúnmente conocido como $\mathrm{MLS}_{\mathrm{B}}$, la cual puede ser constitutiva $\left(\mathrm{CMLS}_{\mathrm{B}}\right)$ o inducible $\left(\right.$ iMLS $\left._{B}\right)$. Estos fenotipos pueden ser identificados por la prueba de doble disco (7).

Estos mecanismos de resistencia se han identificado principalmente en los grupos de clones España ${ }^{6 B_{-}}$ ST90 (8), que se han identificado en Colombia $(9,10)$, y el clon Taiwan ${ }^{23 F}-S T 242$. En un estudio previo realizado en Colombia, se determinó que en los aislamientos de $S$. pneumoniae recuperados

\footnotetext{
Correspondencia:

Marylin Hidalgo, Departamento de Microbiología, Facultad de Ciencias, Pontificia Universidad Javeriana, Carrera 7ํㅡ № 43-82, Bogotá, D.C., Colombia

Teléfono: (571) 320 8320, extensión: 4153.

hidalgo.m@javeriana.edu.co

Recibido: 05/04/10; aceptado:26/08/10
}

de 1994 a 2004, la resistencia a la eritromicina era de $3,3 \%$, y el fenotipo más común fue el cMLSB, el cual estaba asociado con el clon España ${ }^{6 B}$ ST90. En Argentina, además de los clones mencionados, se han identificado los clones Taiwán ${ }^{19 F}$-ST236, Inglaterra ${ }^{14}$-ST9, Polonia ${ }^{6 B_{-}}$ST 315 y España ${ }^{9 V_{-}}$ ST156 (5). Es importante destacar que los serotipos relacionados con estos clones se encuentran incluidos en la vacuna heptavalente $(11,12)$.

El objetivo de este estudio fue determinar la frecuencia de resistencia de $S$. pneumoniae a la eritromicina en Colombia, y caracterizar fenotípica y molecularmente los aislamientos y establecer su relación con los clones internacionales.

\section{Materiales y métodos}

Se analizó la información de todos los aislamientos obtenidos entre 1994 y 2008, en el programa de vigilancia de $S$. pneumoniae del proyecto SIREVA II, de la Organización Panamericana de la Salud que lidera el Grupo de Microbiología del Instituto Nacional de Salud (13-16). Todos los 136 aislamientos fueron recuperados de procesos invasores, de pacientes de todos los grupos de edad y fueron remitidos por los laboratorios de salud pública del país: 52 (38,2 \%) aislamientos provenían de Bogotá, 39 (28,6\%), de Antioquia, $28(20 \%)$, del Valle y 17 (13,2\%), de otros departamentos, así, Risaralda $(n=5)$, Santander $(n=5)$, Caldas $(n=2)$, Cesar $(n=2)$, Bolívar $(n=1)$, Meta $(n=1)$ y Tolima $(n=1)$.

Los datos de los serotipos y de la sensibilidad antimicrobiana se obtuvieron de la base de datos del programa de vigilancia mencionado anteriormente. Los aislamientos habían sido confirmados por técnicas de laboratorio como, aspecto y hemólisis de las colonias, coloración de Gram, sensibilidad a la optoquina y solubilidad en bilis. La serotipificación se hizo con la reacción de Quellung. Todas las técnicas habían sido estandarizadas durante el proyecto SIREVA en el National Centre for Streptococcus (NCS) de Alberta, Canadá (17). Los aislamientos estaban conservados a $-70{ }^{\circ} \mathrm{C}$ en leche descremada al $20 \%$ (Difco). La sensibilidad antimicrobiana se estableció con la determinación de la concentración inhibitoria mínima (CIM) por el método de microdilución en caldo, con la metodología y los parámetros de interpretación del 
Clinical and Laboratory Standards Institute (CLSI) $(18,19)$, a penicilina, eritromicina, cloranfenicol, trimetoprim-sulfametoxazol, tetraciclina, ceftriaxona y vancomicina.

A los aislamientos resistentes a la eritromicina (CIM: $0,5 \mu \mathrm{g} / \mathrm{ml}$ resistencia intermedia y $\mathrm{CIM} \geq 1$ $\mu \mathrm{g} / \mathrm{ml}$, alta resistencia) se les determinó la CIM a clindamicina por el método de microdilución en caldo, con una incubación de 48 horas a $25^{\circ} \mathrm{C}$ (20), y se practicó la prueba de doble disco, para determinar el fenotipo de resistencia, constitutiva $\left(\mathrm{CMLS}_{\mathrm{B}}\right)$, inducible $\left(\mathrm{iMLS}_{\mathrm{B}}\right.$ ) o fenotipo M (21). Además, se hizo la caracterización genotípica, que incluyó detección de los genes ermB y mefA por la técnica de PCR (22) y la determinación del patrón de clones por PFGE, utilizando la enzima de restricción Smal (9). Estos patrones se analizaron visualmente mediante el programa Fingerprinting ${ }^{\circledR}$, versión 2.0, y se interpretaron según los criterios de Tenover (23). Se incluyeron, como controles, S. pneumoniae R6, y los clones España ${ }^{23 F}$ ST81, España $^{6 \mathrm{~B}}$ ST90, España ${ }^{9 \mathrm{~V}}$ ST156 y Colombia ${ }^{23 F}$ ST338.

La información fenotípica y genotípica de los aislamientos resistentes a la eritromicina recolectados de 1994 a 2004, ya había sido publicada (10).

El análisis de los datos se realizó con el programa Epi-Info, versión 6,04. Para determinar el incremento de la resistencia a la eritromicina, se analizaron los datos en períodos de tres años y, para determinar las diferencias estadísticas de las frecuencias, se utilizó el test de Fisher (24).

\section{Resultados}

Se analizaron los datos de 3.248 aislamientos obtenidos en la vigilancia, $1.775(54,6 \%)$ eran de niños menores de 6 años, $315(9,7 \%)$ del grupo de 6 a 14 años y 1.158 (35,7\%) de 15 años o mayores. El número de aislamientos por año y los datos de serotipo y sensibilidad antimicrobiana se encuentran publicados en el portal del internet del Instituto Nacional de Salud (13).
La resistencia a la eritromicina se encontró en 136 $(4,2 \%)$ aislamientos, 76/1.775 (4,8\%) de niños menores de 6 años, 7/315 (2,2\%) de niños de 6 a 14 años y 53/1.158 (4,6\%) de 15 o más años. En el cuadro 1 se consignaron los datos de la resistencia a la eritromicina por grupos de edad y diagnóstico. La resistencia fue mayor en los menores de 6 años y en los de 15 o más años, y en los aislamientos de neumonía y otros diagnósticos $(p<0,001)$ que en los de meningitis.

La distribución de los aislamientos resistentes a la eritromicina, por serotipos, fenotipos y características moleculares, se encuentran en el cuadro 2, al igual que la distribución de serotipos por grupos de edad. En los aislamientos resistentes a la eritromicina, la CIM de la penicilina fue de 0,06 $\mu \mathrm{g} / \mathrm{ml}$ o menor en $15,5 \%$, de 0,125 a $1,0 \mu \mathrm{g} / \mathrm{ml}$ en $36,8 \%$, de $2,0 \mu \mathrm{g} / \mathrm{ml}$ en $30,1 \%$, de $4,0 \mu \mathrm{g} / \mathrm{ml}$ en 16,9 $\%$, y de $8,0 \mu \mathrm{g} / \mathrm{ml}$ en $0,7 \% ; 98$ (72\%) aislamientos eran resistentes a trimetoprim-sulfametoxazol, 78 $(57,4 \%)$, a tetraciclina, $64(47,1 \%)$, a clindamicina y 49 (36\%), a cloranfenicol; todos los aislamientos fueron sensibles a la vancomicina.

En el análisis de los datos del último periodo de estudio (2005-2008) se encontró que, en el 2005, $14 / 286(4,9 \%)$ de los aislamientos habían sido resistentes a eritromicina, en el 2006, 12/299 (4,0\%), en el 2007, 30/351 (8,5\%), y en el 2008, 16/291 (5,5 \%). Al comparar el período 1994-1996 con 2006-2008, se observó un incremento de la resistencia a la eritromicina, que pasó en el total de la población de 2,6 \% a 6,4\% [p<0,01; RR=2,44 $(1,33-4,50)]$, en los niños menores menores de 6 años, de $2,4 \%$ a $6,9 \%$ [p<0,01; RR=2,71 (1,35$5,45)$ ], y en los aislamientos de adultos, de 3,3 $\%$ a $5,7 \%(p=0,4)$ (figura 1$)$. No se analizó el grupo de 6 a 14 años por el número reducido de aislamientos.

El patrón de resistencia, genes y clones se estableció en los 136 aislamientos resistentes a la eritromicina, 87 (64\%), de los cuales, fueron de fenotipo cMLSB; de estos, 82 (94,2\%) expresaron

Cuadro 1. Distribución de la resistencia a eritromicina por grupos de edad y diagnóstico.

\begin{tabular}{lrrrr}
\hline \multirow{2}{*}{ Grupo de edad (años) } & Meningitis & Neumonía & Otros & \multirow{2}{*}{$\mathbf{n}$} \\
\cline { 2 - 3 } & \multicolumn{3}{c}{ Resistencia eritromicina/total (\%) } \\
\hline$<6$ & $16 / 718(2,2)$ & $39 / 702(5,6)$ & $21 / 355(5,9)$ & $76 / 1.775$ \\
6 a 14 & $4 / 138(2,9)$ & $2 / 117(1,7)$ & $1 / 60(1,7)$ & $7 / 315$ \\
$\geq 15$ & $10 / 373(2,7)$ & $27 / 513(5,3)$ & $16 / 272(5,9)$ & $54 / 1.158$ \\
Total & $30 / 1.229(2,4)$ & $68 / 1.332(5,1)$ & $38 / 687(5,3)$ & $136 / 3.248$ \\
\hline
\end{tabular}


Cuadro 2. Distribución de los aislamientos resistentes a eritromicina, por serotipos, fenotipos y características moleculares, Colombia, 1994-2008'.

\begin{tabular}{|c|c|c|c|c|c|c|c|c|c|c|}
\hline \multirow{3}{*}{ Serotipos $^{2}$} & \multirow{3}{*}{$\mathbf{n}$} & \multirow{3}{*}{$(\%)$} & \multicolumn{3}{|c|}{ Fenotipo } & \multirow[t]{3}{*}{ Clon } & & \multicolumn{3}{|c|}{ Gen } \\
\hline & & & cMLSB & $\mathbf{M}$ & iMLSB & & \multirow{2}{*}{$\mathbf{N}$} & ermB & mefA & neg \\
\hline & & & \multicolumn{3}{|c|}{$\mathbf{n}$} & & & \multicolumn{3}{|c|}{$\mathbf{n}$} \\
\hline \multirow[t]{2}{*}{$6 B$} & \multirow{2}{*}{\multicolumn{2}{|c|}{$50(36,8)$}} & 44 & 5 & 1 & España ${ }^{6 B}$ ST90 & 28 & 23 & 4 & 1 \\
\hline & & & & & & No clonal & 22 & 20 & 1 & 1 \\
\hline \multirow[t]{2}{*}{$6 \mathrm{~A}$} & \multirow{2}{*}{\multicolumn{2}{|c|}{$24(17,6)$}} & 3 & 21 & 0 & España ${ }^{6 B}$ ST90 & 1 & 1 & & \\
\hline & & & & & & No clonal & 23 & 2 & 21 & \\
\hline \multirow[t]{2}{*}{14} & \multirow{2}{*}{\multicolumn{2}{|c|}{$23(16,9)$}} & 15 & 7 & 1 & España ${ }^{9 v}$ ST156 & 11 & 7 & 4 & \\
\hline & & & & & & No clonal & 12 & 8 & 3 & 1 \\
\hline $19 \mathrm{~F}$ & 10 & $(7,4)$ & 6 & 4 & 0 & No clonal & 10 & 5 & 4 & 1 \\
\hline \multirow[t]{3}{*}{$19 A$} & 9 & $(6,6)$ & 7 & 2 & 0 & Colombia ${ }^{23 F} S T 338$ & 2 & 1 & 1 & \\
\hline & & & & & & España ${ }^{23 F}$ ST81 & 1 & 0 & 1 & \\
\hline & & & & & & No clonal & 6 & 6 & $1^{3}$ & \\
\hline \multirow[t]{2}{*}{$23 \mathrm{~F}$} & 5 & $(3,8)$ & 5 & 0 & 0 & España $23 F S T 81$ & 3 & 3 & & \\
\hline & & & & & & Colombia ${ }^{23}$ ST338 & 2 & 2 & & \\
\hline \multirow[t]{2}{*}{$9 \mathrm{~V}$} & 3 & $(2,2)$ & 2 & 1 & 0 & España ${ }^{9 v}$ ST156 & 2 & 1 & 1 & \\
\hline & & & & & No clonal & 1 & 1 & & & \\
\hline Otros $^{4}$ & 12 & $(8,7)$ & 5 & 6 & 1 & No clonal & 12 & 4 & 5 & 3 \\
\hline Total & 136 & & & 87 & 46 & 3 & 136 & 84 & 46 & 7 \\
\hline
\end{tabular}

${ }^{1}$ Los datos de 1994-2004 ya fueron publicados (10).

${ }^{2}$ Grupo <6: serotipos: 6B=25 (32,9\%); $14=18(23,7 \%) ; 6 \mathrm{~A}=14(18,4 \%) ; 19 \mathrm{~A}=7$ (9,2\%); $19 \mathrm{~F}=5(6,7 \%) ; 23 \mathrm{~F}=3(3,9 \%) ; 9 \mathrm{~V}=1(1,3 \%)$; 3 eran serotipos $1,18 \mathrm{C}$ y $28 \mathrm{~A}$ (uno de cada uno)

Grupo 6 a 14 años: serotipo $6 \mathrm{~B}=2$; serotipo $6 \mathrm{~A}=2$; 3 eran serotipo 21,14 y $19 \mathrm{~F}$ (uno de cada uno).

Grupo $\geq 15$ serotipos: $6 \mathrm{~B}=23(43,4 \%) ; 6 \mathrm{~A}=8(15,1 \%) ; 14=3(5,7 \%) ; 19 \mathrm{~F}=4(7,5 \%) ; 19 \mathrm{~A}=2(3,8 \%) ; 23 \mathrm{~F}=2(3,8 \%) ; 9 \mathrm{~V}=2(3,8 \%) ; 9$ $(16,9 \%)$ eran de 8 serotipos diferentes.

${ }^{3}$ Aislamiento con dos genes

${ }^{4}$ Otros serotipos, 15A (2); 1, 3, 5, 12B, 16F, 18C, 21, 23B, 28A, 35A (un aislamiento de cada uno)

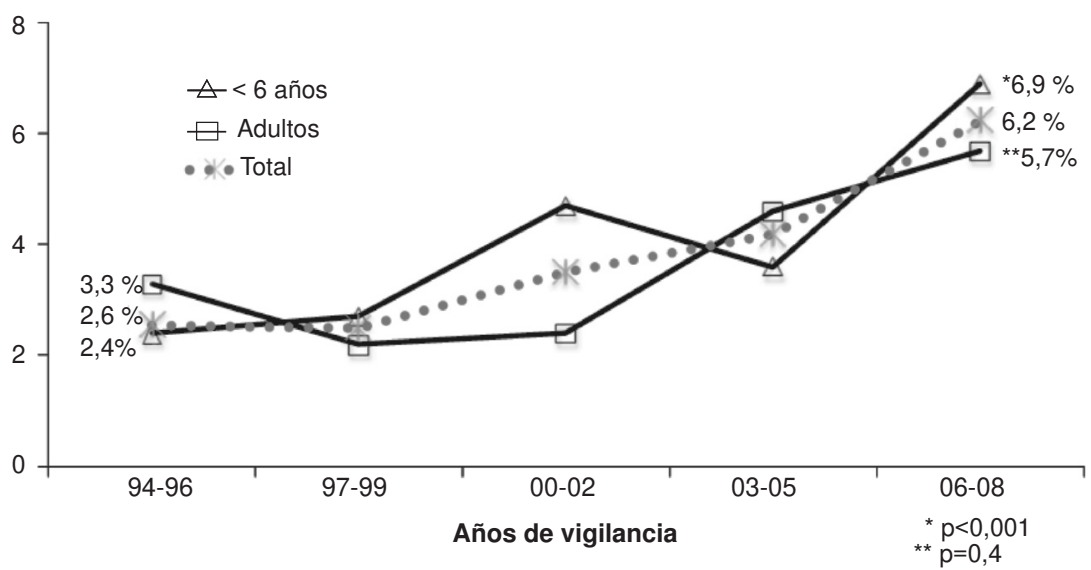

Figura 1. Porcentaje de aislamientos de Streptococcus pneumoniae resistentes a eritromicina por año y grupo de edad, 19942008.

el gen ermB, un aislamiento amplificó el gen mefA y el gen ermB y en 4 no se determinó la presencia de ningún gen. La CIM ${ }_{50}$ y la $\mathrm{CIM}_{90}$ de la clindamicina fueron de $8,0 \mu \mathrm{g} / \mathrm{ml}$ y $32 \mu \mathrm{g} / \mathrm{ml}$, respectivamente, y una $\mathrm{CIM}_{50}$ y una $\mathrm{CIM}_{90}$ de la eritromicina fueron de $32 \mu \mathrm{g} / \mathrm{ml}$. Todos los aislamientos de fenotipo cMLSB fueron multirresistentes, 51/87 $(58,6 \%)$ presentaron el patrón de resistencia a penicilina, eritromicina, cloranfenicol, trimetoprimsulfametozazol, tetraciclina y, de ellos, 56,9 \% tenía resistencia a cloranfenicol.

Cuarenta y seis (34\%) aislamientos eran de fenotipo M, 45 (97,8\%) amplificaron por PCR el gen mefA y uno no presentó ningún gen; tenían una $\mathrm{CIM}_{50}$ y una $\mathrm{CIM}_{90}$ de clindamicina de $0,06 \mu \mathrm{g} /$ $\mathrm{ml}$ y $0,25 \mu \mathrm{g} / \mathrm{ml}$, respectivamente; $20 / 46$ (43,5\%) 
aislamientos presentaron multirresistencia. En el cuadro 3 se encuentran los diferentes perfiles de resistencia.

El fenotipo iMLS $\mathrm{B}_{\mathrm{B}}$ se presentó en tres aislamientos sensibles a la clindamicina, uno tenía el gen ermB y dos no amplificaron ningún gen (emrB o mefA) (cuadro 3).

Con la técnica de PFGE se determinó que, de los 136 aislamientos, 50 (36,7\%) correspondieron a 4 clones internacionales, 29 (58\%) pertenecían al clon España ${ }^{6 \mathrm{~B}}$ ST90, 13 (26\%), al clon España ${ }^{9 \mathrm{~V}}$ ST156, 4 (8\%), al Colombia ${ }^{23 F}$ ST338, y 4 (8\%), al España ${ }^{23 F}$ ST81. En el cuadro 2 están relacionados los clones con el serotipo y la caracterización genotípica. De los 29 aislamientos relacionados con el clon Espana ${ }^{6 B}$ ST90, 17 (58,6 \%) fueron recuperados de neumonía, $6(20,7 \%)$, de meningitis, $4(13,8 \%)$, de sepsis y $2(6,8 \%)$ no tenían dato. Del total de los aislamientos correspondían a este clon, $16 / 76(14,5 \%)$ de los menores de 6 años, $2 / 7$ (28,6 $\%)$ de los niños de 6 a 14 años y 11/54 (20,4\%) de los pacientes mayores de 15 años.

\section{Discusión}

El análisis de la información obtenida en la vigilancia que se realiza con el proyecto SIREVAOPS determinó el incremento en la resistencia a la eritromicina de los aislamientos invasores colombianos de $S$. pneumoniae. Esta información confirma la importancia de realizar este tipo de vigilancia (13-16).

De acuerdo con los resultados de la vigilancia de SIREVA II en 20 países latinoamericanos, publicados por la OPS (14), en el periodo 20002005 México, Venezuela y Nicaragua presentaron alta resistencia a la eritromicina en 37,1 \%, 31,7 \% y $20,5 \%$ de los casos, respectivamente; Colombia

Cuadro 3. Asociación de los patrones de resistencia con los fenotipos y los clones internacionales, Colombia, 1994-2008.

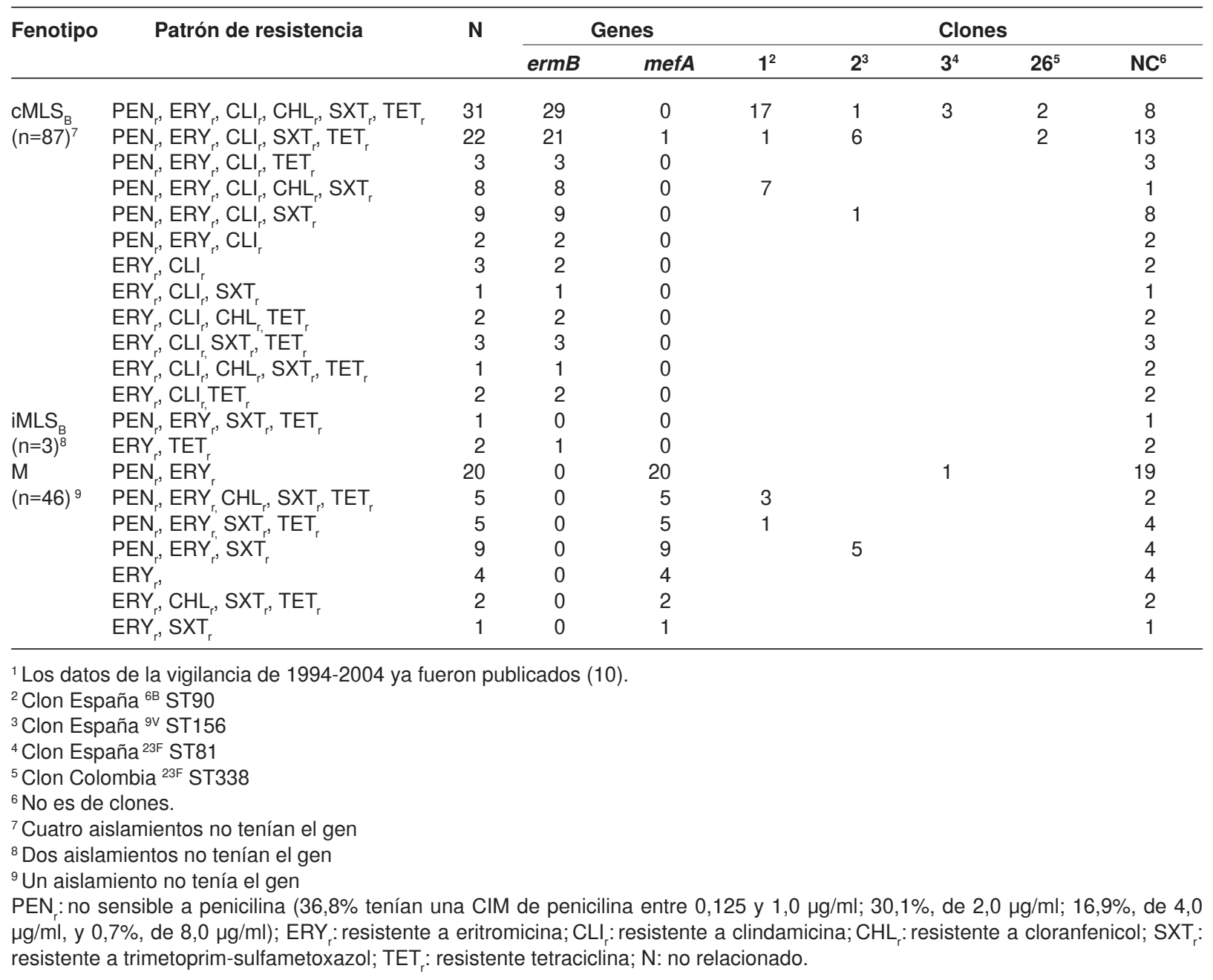


tenía una de las resistencias (4,4 \%) más bajas de los países de la región, pero en este estudio se encontró que en Colombia hay una clara tendencia al incremento de la resistencia, en especial, en los aislamientos de niños menores de 6 años, ya que entre 2006 y 2008 la resistencia fue de 6,9 $\%$ (figura 1). En este estudio los serotipos más frecuentemente relacionados con resistencia a la eritromicina fueron el 6B, $6 \mathrm{~A} / \mathrm{C}, 14,19 \mathrm{~F}$ y $19 \mathrm{~A}$, similares a los que circulan en Europa, donde los más importantes son el 14,6B, 23F, 19F y $19 \mathrm{~A}$ (25) y, con menos frecuencia, 15A, 15F y 38 , los cuales no han sido reportados en Colombia. Todos los serotipos mencionados se han asociado con clones de distribución mundial.

La presencia de aislamientos con perfiles de multirresistencia puede deberse a la presencia de transposones de la familia $\mathrm{Tn} 916$ relacionados principalmente con el fenotipo $\mathrm{MLS}_{\mathrm{B}}$, y con el Tn1207.1. asociado con el fenotipo M; aunque la presencia de estos transposones no se estableció en este estudio, puede ser útil para determinar la transferencia horizontal de este mecanismo de resistencia (26).

El fenotipo más frecuentemente identificado fue el cMLS, similar a los resultados del estudio realizado en Colombia en el año 2004 (10) y a los análisis hechos en Francia, España, Suiza y Polonia $(21,27)$, mientras que en otros países, como Grecia, Alemania, Chile y un estudio reciente publicado en Argentina, el fenotipo $\mathrm{M}$ es el más importante $(5,25,28,29)$.

Algunos estudios publicados han mostrado que los aislamientos de $S$. pneumoniae con una $\mathrm{CIM}_{90}$ de la eritromicina de $32 \mu \mathrm{g} / \mathrm{ml}$ o mayor, están asociados con la presencia del gen ermB, lo cual concuerda con los resultados obtenidos en este análisis (30). La $\mathrm{CIM}_{90}$ de 1 a $4 \mu \mathrm{g} / \mathrm{ml}$ de la eritromicina está relacionada con el gen mefA; sin embargo, datos recientes muestran que hay un incremento en la proporción de aislamientos que expresan el gen mefA y tienen una $\mathrm{CIM}_{90}$ de $16 \mu \mathrm{g} / \mathrm{ml}$ (4), lo que explica lo encontrado por nosotros, que los aislamientos con el gen mefA presentaron una $\mathrm{CIM}_{90}$ de $8,0 \mu \mathrm{g} / \mathrm{ml}$. Por otro lado, es importante tener en cuenta que $61,8 \%$ de los aislamientos colombianos de este estudio portan el gen ermB, datos similares a los de países europeos (25) y a estudios realizados en Brasil (31). Esto demuestra que los aislamientos colombianos presentan mayor resistencia a la eritromicina, lo que se refleja en una CIM más alta.
En nuestro estudio se encontraron tres aislamientos con el fenotipo $\mathrm{iMLS}_{\mathrm{B}}$; a pesar de no ser tan frecuente, este fenotipo se ha reportado en varios estudios. En 2007, Catalayud et al. reportaron que, de 125 aislamientos de S. pneumoniae resistente a la eritromicina, $18(14,4 \%)$ expresaron el fenotipo iMLSB, 18 tenían el gen ermB y uno de ellos portaba el gen mefE (27). En nuestros aislamientos con fenotipo inducible, uno de los aislamientos tenía el gen ermB. Otro estudio publicado en el 2003 mostró que el fenotipo inducible estuvo presente en $3,8 \%$ de los aislamientos estudiados (32).

En los otros dos aislamientos con fenotipo inducible, al igual que con los cuatro aislamientos que expresaron el fenotipo constitutivo y un aislamiento que expresó el fenotipo $\mathrm{M}$, no se detectó ninguno de los dos genes estudiados, mefA y ermB. Es probable que estos aislamientos tengan otros genes que codifiquen para metilasas o que tengan mecanismos menos comunes, como son las mutaciones en el 23S RNAr o alteraciones en la riboproteínas L4 o L22 $(33,34)$.

Eneste estudio, el clon España ${ }^{6 \mathrm{~B}}{ }_{-S T}$ ST0 y el España ${ }^{9 V_{-}}$ ST156 se identificaron con mayor frecuencia $(21,3$ \% y 9,6 \%), a diferencia de países como España, donde los principales clones relacionados con la resistencia a macrólidos es el España ${ }^{23 F}$-ST81 y el Suecia ${ }^{15 A}$-ST63, con 14,3 \% cada uno. Esto se relaciona con los serotipos que son más frecuentes en cada uno de los países (27).

La relación de clones de algunos aislamientos de los serotipos 6A, 14 y $19 \mathrm{~A}$ con clones como España $^{6 \mathrm{~B}}$ ST90 y el España ${ }^{9 \mathrm{~V}}$ ST156 y Colombia ${ }^{23 F}$ ST338, puede explicarse en el contexto del recambio (switching) capsular, para el cual existen diferentes hipótesis, entre las cuales se encuentran: tipos capsulares con éxito como colonizadores, determinantes de virulencia asociados con determinados tipos capsulares $\mathrm{y}$, actualmente, cambios de serotipos de vacuna por de no vacuna como consecuencia de la presión ejercida por la vacunación (35). En este estudio, el cambio capsular se asoció a serotipos con alta resistencia a la eritromicina.

La información generada en este análisis demuestra la necesidad de mantener este tipo de vigilancia por el laboratorio, la cual permitió demostrar el incremento de la resistencia a la eritromicina y, además caracterizar, los aislamientos. Esto también ya ha sido demostrado en otros estudios, como el realizado recientemente en Estados Unidos que reveló la importancia de la caracterización tanto 
fenotípica como genotípica de los aislamientos resistentes a la eritromicina, ya que se han incrementado los aislamientos de $S$. pneumoniae con CIM altas que puedan portar los dos genes (ermBy mefA) simultáneamente (4).

\section{Agradecimientos}

A los coordinadores de los laboratorios de salud pública que, a través de la Red Nacional de Laboratorios, enviaron los aislamientos dentro de los programas de vigilancia de neumonías y meningitis bacterianas.

A Jaime Moreno, María Victoria Ovalle, Olga Marina Sanabria y Catering Rodríguez, del Grupo de Microbiología del Instituto Nacional de Salud.

\section{Conflicto de intereses}

Los autores declaran no tener ningún conflicto de intereses.

\section{Financiación}

Este trabajo fue financiado por el Instituto Nacional de Salud.

\section{Referencias}

1. Musher DM. Infections caused by Streptococcus pneumoniae: Clinical spectrum, pathogenesis, immunity, and treatment. Clin Infect Dis. 1992;14:801-7.

2. Van Bambeke F, Reinert RR, Appelbaum PC, Tulkens PM, Peetermans WE. Multidrug-resistant Streptococcus pneumoniae infections: Current and future therapeutic options. Drugs. 2007;67:2355-82.

3. Lonks JR, Garau J, Gómez L, Xercavins M, Ochoa de Echagüen A, Gareen IF, et al. Failure of macrolide antibiotic treatment in patients with bacteremia due to erythromycinresistant Streptococcus pneumoniae. Clin Infect Dis. 2002;35:556-64.

4. Jenkins SG, Farrell DJ. Increase in pneumococcus macrolide resistance, United States. Emerg Infect Dis. 2009;15:1260-4.

5. Corso A, Faccone C, Galiá P, Gagetti M, Rodríguez $\mathbf{J}$, Pace M. et al. Prevalence of mef and ermB genes in invasive pediatric erythromycin-resistant Streptococcus pneumoniae isolates from Argentina. Rev Argent Microbiol. 2009;41:29-33.

6. Leclercq R, Courvalin P. Resistance to macrolides and related antibiotics in Streptococcus pneumoniae. Antimicrob Agents Chemother. 2002;46:2727-34.

7. Marimón JM, Iglesias L, Vicente D, Pérez-Trallero E. Molecular characterization of erythromycin-resistant clinical isolates of the four major antimicrobial-resistant Spanish clones of Streptococcus pneumoniae (Spain23F-1, Spain6B-2, Spain9V-3, and Spain14-5). Microb Drug Resist. 2003;9:133-7.

8. Chiou CC, McEllistrem MC. Novel penicillin, cephalosporin, and macrolide resistant clones of Streptococcus pneumoniae serotypes $23 \mathrm{~F}$ and $19 \mathrm{~F}$ in Taiwan which differ from international epidemic clones. J Clin Microbiol. $2001 ; 39: 1144-7$

9. Vela MC, Fonseca N, Di Fabio JL, Castañeda E. Presence of international multiresistant clones of Streptococcus pneumoniae in Colombia. Microb Drug Resist. 2001; 7:153-64.

10. Reyes J, Hidalgo M, Díaz L, Rincón S, Moreno J, Vanegas $\mathbf{N}$, et al. Characterization of macrolide resistance in Grampositive cocci from Colombian hospitals: A countrywide surveillance. Int J Infect Dis. 2007;11:329-36.

11. De la Pedrosa EG, Morosini MI, van der Linden M, RuizGarbajosa P, Galán JC, Baquero F, et al. Polyclonal population structure of Streptococcus pneumoniae isolates in Spain carrying mef and mef plus erm(B). Antimicrob Agents Chemother. 2008;52:1964-9.

12. Whitney C, Pilishvili T, Farley M, Schaff ner W, Craig A, Lynfield R, et al. Effectiveness of seven-valent pneumococcal conjugate vaccine against invasive pneumococcal disease: a matched case-control study. Lancet. 2006;368:1495-502.

13. Instituto Nacional de Salud. Serotipos y patrones de susceptibilidad antimicrobiana de patógenos de importancia en salud pública. Fecha de consulta: 31 de julio de 2009. Disponible en: http://www.ins.gov.co/?idcategoria=6134\#.

14. Organización Panamericana de la Salud. Informe Regional de SIREVA II: Datos por país y por grupo de edad sobre las características de los aislamientos de Streptococcus pneumoniae, Haemophilus influenzae y Neisseria meningitidis en procesos invasores 2000-2005. Washington, D.C.: OPS; 2007. (THS/EV - 2007/002:1-387). Fecha de consulta: 31 de julio de 2009. Disponible en: http:// www.paho.org/Spanish/AD/THS/EV/LABS-Sireva.pdf.

15. Organización Panamericana de la Salud. Informe regional de SIREVA II, 2006: datos por país y por grupos de edad sobre las características de los aislamientos de Streptococcus pneumoniae, Haemophilus influenzae y Neisseria meningitidis, en procesos invasores. Washington, D.C.: OPS; 2008. (THR/EV - 2008/001:1-288). Fecha de consulta: 31 de julio de 2009. Disponible en: http://www. paho.org/Spanish/AD/THS/EV/labs_Sireva_II_2006.pdf.

16. Organización Panamericana de la Salud. Informe regional de SIREVA II, 2007: datos por país y por grupos de edad sobre las características de los aislamientos de Streptococcus pneumoniae, Haemophilus influenzae y Neisseria meningitidis, en procesos invasores. Washington, D.C.: OPS; 2008. (THR/EV - 2008/003:1-310). Fecha de consulta: 31 de julio de 2009. Disponible en: http://new. paho.org/hq/index.php?option=com_content\&task=view\&id $=1077$ \&ltemid=591\&limit=1\&limitstart=2.

17. Organización Panamericana de la Salud e Instituto Nacional de Salud Colombia. Programa de vigilancia de los serotipos y resistencia antimicrobiana de Streptococcus pneumoniae y Haemophilus influenzae. Manual de procedimientos del proyecto SIREVA II. 2004. p. 41-99. Fecha de consulta: 9 de diciembre de 2008. Disponible en: http://www.paho.org/Spanish/AD/THS/EV/LABS-manualvigilancia-serotipos.pdf.

18. Clinical and Laboratory Standards Institute. Methods for dilution antimicrobial susceptibility tests for bacteria that grow aerobically; approved standard-7th ed. Document M7-A7. 
Wayne, PA: Clinical and Laboratory Standards Institute; 2006.

19. Clinical and Laboratory Standards Institute. Performance standards for antimicrobial susceptibility testing, 16th informational supplement CLSI document M100-S18 2008. Wayne, PA: Clinical and Laboratory Standards Institute; 2008.

20. Fasola EL, Bajaksouzian S, Appelbaum PC, Jacobs MR. Variation in erythromycin and clindamycin susceptibilities of Streptococcus pneumoniae by four test methods. Antimicrob Agents Chemother. 1997;41:129-34.

21. Montanari MP, Mingoia M, Giovanetti, Varaldo PE. Differentiation of resistance phenotypes among erythromycin-resistant pneumococci. J Clin Microbiol. 2001; 39:1311-5.

22. Sutcliffe J, Grebe T, Tait-Kamradt A, Wondrack L. Detection of erythromycin-resistant determinants by PCR. Antimicrob Agents Chemother. 1996;40:2562-6.

23. Tenover FC, Arbeit RD, Goering RV, Mickelsen PA, Murray BE, Persing DH, et al. Interpreting chromosomal DNA restriction patterns produced by pulsed-field gel electrophoresis: criteria for bacterial strain typing. J Clin Microbiol. 1995;33:2233-9.

24. Dean AG, Dean JA, Columbier D, Burton AH, Brendel KA, Smith DC, et al. Epi Info versión 6.0: A word processing, data-base and statistics program for epidemiology on microcomputers. Atlanta, Georgia: Centers for Disease Control and Prevention; 1994.

25. Reinert RR, Ringelstein A, van der Linden M, Cil MY, Al-Lahham A, Schmitz FJ. Molecular epidemiology of macrolide-resistant Streptococcus pneumoniae isolates in Europe. J Clin Microbiol. 2005;43:1294-300.

26. Xu X, Cai L, Xiao M, Kong F, Oftadeh S, Zhou F, et al. Distribution of serotypes, genotypes, and resistance determinants among macrolide-resistant Streptococcus pneumoniae isolates. Antimicrob Agents Chemother. 2010;54:1152-9.
27. Calatayud L, Ardanuy C, Cercenado E, Fenoll A, Bouza E, Pallares R, et al. Serotypes, clones, and mechanisms of resistance of erythromycin-resistant Streptococcus pneumoniae isolates collected in Spain. Antimicrob Agents Chemother. 2007;51:3240-6.

28. van der Linden M, Al-Lahham A, Haupts S, Reinert RR. Clonal spread of mef-positive macrolide-resistant Streptococcus pneumoniae isolates causing invasive disease in adults in Germany. Antimicrob Agents Chemother. 2007:51:1830-4

29. Palavecino EL, Riedel I, Duran C, Bajaksouzian S, Joloba M, Davies T, et al. Macrolide resistance phenotypes in Streptococcus pneumoniae in Santiago, Chile. Int J Antimicrob Agents. 2002;20:108-12.

30. Farrell DJ, File TM, Jenkins SG. Prevalence and antibacterial susceptibility of $\operatorname{mef}(\mathrm{A})$-positive macrolideresistant Streptococcus pneumoniae over 4 years $(2000$ to 2004) of the PROTEKT US Study. J Clin Microbiol. 2007;45:290-3.

31. Mendonça-Souza CR, Carvalho M da G, Barros RR, Dias CA, Sampaio JL, Castro AC, et al. Occurrence and characteristics of erythromycin-resistant Streptococcus pneumoniae strains isolated in three major Brazilian states. Microb Drug Resist. 2004;10:313-20.

32. Melo-Cristino J, Ramírez M, Serrano N, Hänscheid T. Resistance in Streptococcus pneumoniae isolated from patients with community-acquired lower respiratory tract infections in Portugal: Results of a 3-Year (1999-2001). Microb Drug Resist. 2003;9:73-80.

33. Weisblum B. Erythromycin resistance by ribosome modification. Antimicrob Agents Chemother 1995;39:577-85.

34. Roberts MC. Update on macrolide-lincosamidestreptogramin, ketolide, and oxazolidinone resistance genes. FEMS Microbiol Lett. 2008;282:147-59.

35. Temime L, Boelle PY, Opatowski L, Guillemot D. Impact of capsular switch on invasive pneumococcal disease incidence in a vaccinated population. PLoS One. 2008;3:e3244. 